\title{
Osteonecrosis vertebral y vertebroplastia percutánea
}

\author{
Lluís Rossellóa, Francesc Pallisóa, Jesús Ferrer ${ }^{b}$, Elisa Docampoc, Joan Calvet ${ }^{c}$, Pere Benito ${ }^{c}$ \\ y Joan Serrad \\ aUnidad de Reumatología y Aparato Locomotor. Hospital de Santa María. Lleida. España. \\ bervicio de Radiología. Clínica de Ponent. Lleida. España. \\ 'Servicio de Reumatología. IMAS. Hospital del Mar. Barcelona. España. \\ dServico de Radiología. CRC. Hospital Universitario de Bellvitge. L’Hospitalet del Llobregat. Barcelona. España.
}

La osteonecrosis vertebral se caracteriza por presentar el fenómeno de vacío intravertebral. Es un proceso poco frecuente y aunque puede ser debido a diferentes afecciones, la causa más frecuente es la postraumática. La explicación de que aparezca gas intravertebral no es del todo conocida. Presentamos el caso de una paciente de 74 años que después de sufrir un traumatismo vertebral inició clínica de dolor dorsolumbar intenso. El estudio radiológico simple, la tomografía computarizada y la resonancia magnética confirmaron el fenómeno del vacío intravertebral. Hemos hecho una revisión de este signo radiológico y comentamos la evolución después de vertebroplastia percutánea.

Palabras clave: Fracturas osteoporóticas. Osteonecrosis vertebral. Vertebroplastia percutánea.

\section{Vertebral Osteonecrosis and Percutaneous Vertebroplasty}

Vertebral osteonecrosis is characterized by the presence of the intravertebral vacuum phenomenon. It is a relatively uncommon disease and although it may be caused by different pathologies, the most frequent cause is posttraumatic. The explanation for the presence of intravertebral gas is not known completely. We present the case of a 74-year-old patient who after suffering a vertebral traumatism, to complain of intense vertebral pain. A simple radiological study, CT scan, and magnetic resonance confirmed the presence of intravertebral vacuum phenomenon. We studied this radiological sign and then commented on its evolution after percutaneous vertebroplasty.

\footnotetext{
Correspondencia: Dr. L. Roselló Aubach.

Unidad de Reumatología y Aparato Locomotor. Hospital de Santa María. Avda. Rovira Roure, 44. 25198 Lleida. España.

Correo electrónico: Irosello@gss.scs.es
}

Manuscrito recibido el 30-5-2007 y aceptado el 24-10-2007.
Key words: Osteoporotic fractures. Vertebral osteonecrosis. Percutaneous vertebroplasty.

\section{Introducción}

El fenómeno del vacío intravertebral (FVI) es un signo radiológico muy poco frecuente $\mathrm{y}$, aunque no patognomónico, es la característica más relevante de la osteonecrosis vertebral ${ }^{1}$. No fue hasta el año 1979 en que Maldague et $\mathrm{al}^{2}$ asociaron esta presencia de gas con la isquemia del cuerpo vertebral. A pesar de haber algunos casos descritos de osteonecrosis sin colapso vertebral ${ }^{3}$, en la mayoría de las ocasiones el fenómeno del vacío intravertebral se localiza en una vértebra fracturada y colapsada, especialmente en gente mayor y con osteoporosis previa, aunque el mecanismo exacto de la formación de gas intravertebral es desconocido. El propósito de este artículo es presentar el caso de una mujer con fractura traumática vertebral que desarrolló FVI y adicionalmente hemos realizado una revisión de la literatura al respecto, y finalmente se comenta la progresión del caso después de vertebroplastia percutánea.

\section{Caso clínico}

Mujer de 74 años de edad, con antecedentes personales de hipertensión arterial tratada con atenolol $100 \mathrm{mg} /$ día y síndrome depresivo en tratamiento con venlafaxina 75 $\mathrm{mg} /$ día, diagnosticada de osteoporosis 10 años antes con fractura de Colles bilateral hacía 4 años y varias fracturas vertebrales postraumáticas (D9, L2 y L3), en tratamiento con $2 \mathrm{~g}$ al día de ranelato de estroncio, $1.000 \mathrm{mg}$ al día de calcio y $400 \mathrm{U}$ al día de vitamina D. $\mathrm{Su}$ estado general era aceptable, cuando en noviembre de 2006 sufrió una aparatosa caída casual sobre su espalda por lo que quedó prácticamente inmovilizada por el dolor intenso en la columna dorsolumbar. Fue trasladada a urgencias del hospital de referencia donde se le practicó analítica y radiología vertebral y con el diagnóstico de fracturas vertebrales recientes y antiguas fue 




Figura 1. Imagen de la tomografía computarizada que muestra vacío intravertebral en la vértebra dorsal 12.

dada de alta al domicilio; se le recomendó reposo absoluto y antiálgicos (paracetamol $1 \mathrm{~g} / 8 \mathrm{~h}$, tramadol 150 $\mathrm{mg} / 12 \mathrm{~h}$ e ibuprofeno $600 \mathrm{mg} / 12 \mathrm{~h}$ ). Se cambió el tratamiento de ranelato de estroncio por teriparatida subcutánea y 1 mes después se trasladó a la paciente para completar estudio analítico y radiológico por persistencia de intenso dolor dorsolumbar, limitación grave de la movilidad e intolerancia a la deambulación. En la analítica destacaba: urea, 61 (valores normales, 10-50) $\mathrm{mg} / \mathrm{dl}$; ácido úrico, 7,5 (2,4-6) mg/dl; colesterol, 260 (150-220) mg/dl; ALT, 37 (5-31) U/l; ferritina, 311,5 (30-300) ng/ml; PCR, 8,6 (0,01-6) mg/1, y PTH intacta, 2,2 (1,6-6,9) pmol/1. El resto de la analítica: hemograma, VSG, proteinograma, hormonas tiroideas, metabolismo fosfocálcico, calciuria y fosfaturia $24 \mathrm{~h}, \mathrm{CEA}$, vitamina D3 y telopéptidos (NTX y CTX) estaban dentro de la normalidad. Durante una semana y por este orden se realizaron las pruebas de imagen: radiografía simple de columna dorsolumbar: fractura-aplastamiento vertebral en D9, D11, L1, L2 y L3. Tomografía computarizada (TC) de columna dorsal: fractura-aplastamiento en D12 con fenómeno de vacío intravertebral muy indicativo de osteonecrosis (fig. 1), aplastamientos vertebrales en D9, D11, L2 y L3. Gammagrafía ósea con ${ }^{99 \mathrm{~m}} \mathrm{Tc}$ c: reacción osteogénica intensa en cuerpo vertebral de D12. Resonancia magnética (RM) dorsolumbar: fracturas osteoporóticas antiguas en grado moderado en D9, L1 y graves en D11 y L3. En D12 fractura osteoporótica de grado moderado, aguda-subaguda, con disminución difusa de la señal en secuencias T1 y un aumento difuso de la señal en secuencia poten- ciada en T2 con supresión grasa (STIR). Centralmente, imagen de vacío de señal en ambas secuencias, secundaria a aire intravertebral y foco de intensidad líquida en STIR muy indicativo de osteonecrosis (fig. 2). Por la persistencia de la dorsolumbalgia invalidante y ante las imágenes radiológicas se consultó el caso con el Servicio de Radiología y Reumatología del IMAS Hospital del Mar de Barcelona y la paciente ingresó, 2 meses después del traumatismo, para vertebroplastia de vértebra D12 que se realizó por vía yuxtapedicular derecha con 2-3 ml de metilmetacrilato y también de cuerpo vertebral de D11 que presentaba aplastamiento antiguo, pero con cierto edema posterior también indicativo de necrosis vertebral, igualmente por vía yuxtapedicular derecha y con $2 \mathrm{ml}$ de metilmetacrilato. La tolerancia al procedimiento fue buena y la respuesta al dolor, inmediata (EVA previo, 8/10; EVA posvertebroplastia, 3/10). Un mes después de la intervención la paciente seguía con cierta limitación a la movilidad dorsolumbar y el único tratamiento que recibía era teriparatida subcutánea y $2.000 \mathrm{mg}$ diarios de calcio con $800 \mathrm{U}$ de vitamina $\mathrm{D}$ sin ningún tipo de analgesia, con un EVA de 2/10. En la figura 3 se muestra la radiografía simple de columna dorsal, 6 semanas después de la vertebroplastia.

\section{Discusión}

El FVI revela la presencia de gas dentro del hueso. Suele estar compuesto por nitrógeno en un $95 \%$ y, en menor cantidad, oxígeno y dióxido de nitrógeno. Desde la

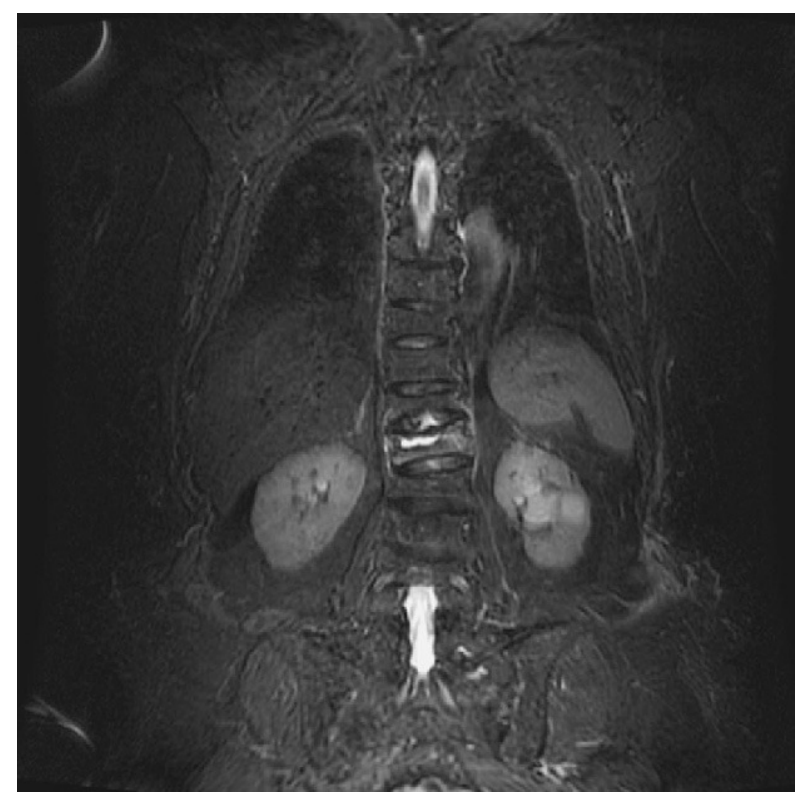

Figura 2. Imagen de la resonancia magnética en fase de secuencia de supresión grasa. Puede verse la imagen de vacío intravertebral y ocupación con edema óseo de la vértebra dorsal 12. 


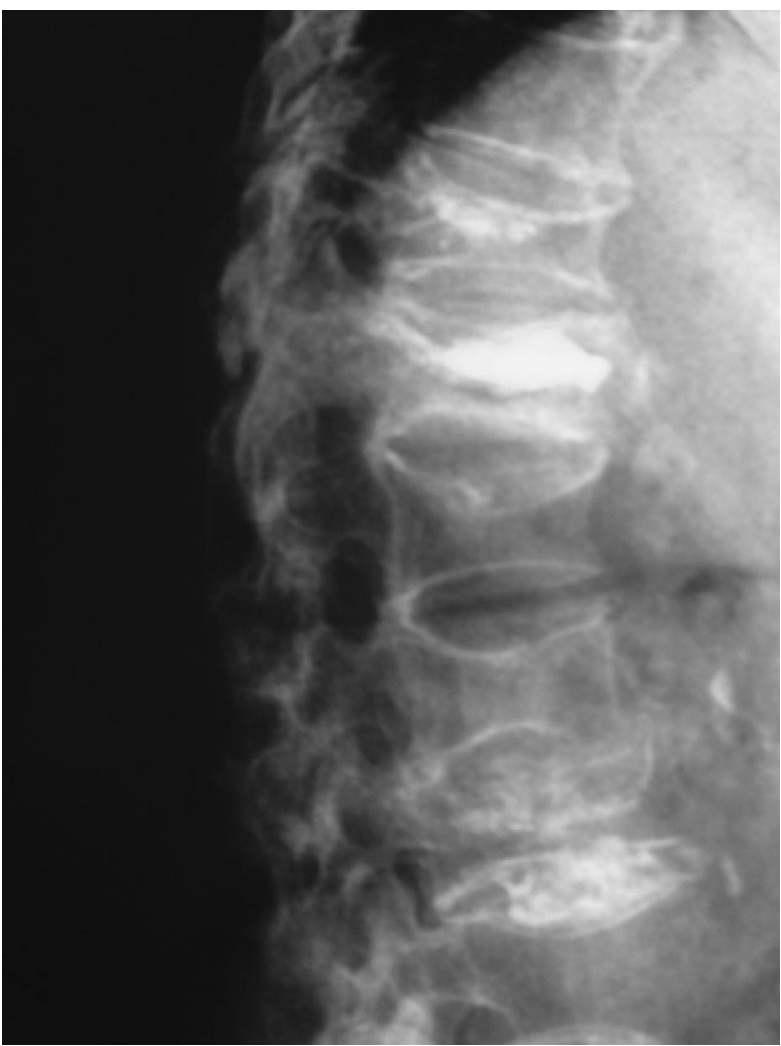

Figura 3. Imagen radiográfica de vertebroplastia D12 y D11.

descripción de Maldague et $\mathrm{al}^{2}$, se admite que la fisiopatología del FVI es atribuida a una osteonecrosis vertebral, relativamente frecuente en gente mayor y con osteoporosis, en los que hay inadecuada vascularización vertebral, error en el proceso reparativo y alteración en la cicatrización, aunque el mecanismo de la formación de este gas intravertebral permanece en hipótesis. La frecuente observación de gas discal adyacente a un FVI ha hecho proponer la hipótesis de una posible migración de gas proveniente de un disco contiguo a través de una plataforma vertebral fracturada ${ }^{4,5}$. También se ha propuesto que podría ser resultado de la vaporización y la acumulación en la grieta del gas soluble que permanece disuelto en los tejidos que envuelven a la vértebra ${ }^{6}$, o mucho más raro, y sin tener relación con el caso que aportamos, la producción de gas intravertebral podría estar en relación con osteomielitis causada por bacterias formadoras de $\mathrm{gas}^{7}$.

El FVI puede observarse en una radiografía anteroposterior y lateral de columna en forma de una imagen lineal o en media luna, transparente, y que, curiosamente, puede intensificarse en extensión de la columna o desaparecer en flexión. En imagen con TC la colección gaseosa muestra una forma más homogénea e irregular que la radiografía. En el caso que presentamos la radiografía inicial de columna dorsal no hacía sospechar la osteonecrosis al no verse el FVI, en cambio sí se detectó con TC 1 mes después del traumatismo, esto quizá fuera debido a que el hematoma intraóseo, que se da en el colapso vertebral agudo, ocupa las hendiduras entre los fragmentos óseos e impide la colección de gas entre estas hendiduras. Tanto la gammagrafía ósea, en la que se observa un aumento inespecífico de captación de la vértebra afectada, como la RM, ayudan a confirmar la localización. Con la RM en secuencia T1 se ve una imagen de baja intensidad de densidad aire, pero en T2 esta intensidad depende del tiempo y la posición del paciente: en los primeros 10 min desde la posición supina las imágenes que se ven son de baja intensidad, entre 20 y $40 \mathrm{~min}$ la intensidad es marcadamente alta, por contenido fluido que indica la afluencia lenta de líquido dentro de la grieta ${ }^{7}$. Estos cambios de señal indican que estamos ante un proceso agudo susceptible de tratamiento con vertebroplastia.

El tratamiento de la osteonecrosis vertebral es el mismo que el de la fractura vertebral osteoporótica, reposo, analgesia e inmovilización, y si en el transcurso de 4 o 6 semanas persiste el dolor, y descartadas complicaciones neurológicas, musculares u ortopédicas, deben considerarse tratamientos más agresivos como la vertebroplastia o la cifoplastia ${ }^{8}$. La paciente del caso que presentamos no mejoró después del primer mes de tratamiento no intervencionista y, ante el riesgo de colapso vertebral severo, se decidió realizar vertebroplastia percutánea. Esta técnica consiste en la inyección percutánea de polimetilmetacrilato dentro del cuerpo vertebral de la vértebra osteonecrótica con una cánula por vía pedicular, yuxtapedicular o posterolateral, con guía fluoroscópica o TC. Finalizado el procedimiento se retiran las agujas, se mantiene al paciente en decúbito durante 1 o $2 \mathrm{~h}$ y se le da de alta. El alivio del dolor puede apreciarse generalmente entre 2 y 24 h después de la intervención, como ocurrió en nuestra paciente. La mayoría de las series publicadas hasta la fecha suelen ser de estudios retrospectivos y coinciden en una respuesta terapéutica funcional del orden del 90\%, el número de vértebras tratadas varía entre 1 y 3 por paciente y la tasa de complicaciones posterapéuticas, como extravasación de cemento, hemorragias u otras, se estima en menos del $10 \%{ }^{9-11}$. Estudios prospectivos han demostrado mejoría en la alineación de la columna de los pacientes tratados por corrección de cifosis o restauración de la altura del cuerpo vertebra1 ${ }^{12-14}$. Algunos trabajos ${ }^{15,16}$ han demostrado, sin embargo, un alto riesgo de nuevas fracturas, que entre el 40 y el $50 \%$ se dan en vértebras adyacentes a la vertebroplastia y más precoces que otras fracturas, lo que hace pensar en algún conflicto mecánico entre la vértebra osteoporótica y la cimentada.

Un mes después de la intervención la paciente que presentamos permanecía con cierta limitación a la funcionalidad, pero no tomaba antiálgicos y mostraba un alto grado de satisfacción. 


\section{Agradecimientos}

Aprovechamos la publicación de este caso clínico para recordar a la Dra. Mónica Sanmartí García, autora de una de las mejores revisiones sobre la osteonecrosis vertebral $^{1}$ y que durante el tiempo que estuvo trabajando con nosotros (Clínica de Ponent) dio muestras de gran profesionalidad y compañerismo.

\section{Bibliografía}

1. Sanmartí M. Osteonecrosis vertebral. Seminarios de la Fundación Española de Reumatología. 2004;5:248-53.

2. Maldague BE, Noel HM, Malghem JJ. The intravertebral vacuum cleft: a sign of ischemic vertebral collapse. Radiology. 1978;129:23-9.

3. Murakami H, Kawahara N, Gabata T, Nambu K, Tomita K. Vertebral body osteonecrosis without vertebral collapse. Spine. 2003;16:323-8.

4. Lafforgue P, Chagnaud C, Daumen-Legre V, Daver L, Kasbarian M, Acquaviva $\mathrm{P}$. The intravertebral vacuum phenomenon ("vertebral osteonecrosis"). Migration of intradiscal gas in fractured vertebral body ? Spine. 1997;22:1885-91

5. Armingeat T, Pham T, Legre V, Lafforgue P. Coexistence of intravertebral vacuum and intradiscal vacuum. Joint Bone Spine. 2006;73:42832 .
6. Theodorou DJ. The intravetebral vacuum cleft sign. Radiology. 2001;221: 787-8.

7. Sarli M, Pérez FC, Gallo R, Zanchetta JR. The vacuum cleft sign: an uncommon radiological sign. Osteoporos Int. 2005;16:1210-4.

8. Mathis JM. Vertebroplasty versus kyphoplasty: a comparison and contrast. Am J Neuroradiol. 2004;25:840-5.

9. Evans AJ, Jensen ME, Kip KE, DeNardo AJ, Lawler GJ, Negin GA. Vertebral compression fractures: pain reduction and improvement in functional mobility after percutaneous polymethylmethacrylate vertebroplasty -retrospective report of 245 cases. Radiology. 2003;226:366-72.

10. Watts NB, Harris ST, Genant HK. Treatment of painful osteoporotic vertebral fractures with percutaneous vertebroplasty or kyphoplasty. Osteopor Int. 2001;12:429-37.

11. Peh WC, Gilula LA, Peck DD. Percutaneous vertebroplasty for severe osteoporotic vertebral compression fractures. Radiology. 2002;223:121-6.

12. Hiwatashia A, Moritania T, Numaguchia Y, Westessona PL. Increase in vertebral body height after vertebroplasty. Am J Neuroradiol. 2003;24: 185-9.

13. Chang C, Teng MM, Wei C, Luo C, Chang F. Percutaneous vertebroplasty for patients with osteoporosis: a one-year follow-up. Acta Radiol. 2006;47:568-73.

14. McKiernan F, Jensen R, Faciszewski T. The dynamic mobility of vertebral compression fractures. J Bone Min Res. 2003;18:24-9.

15. Voormolen NH, Lohle PN, Juttmann JR, Graaf Y, Fransen H, Lampmann LE. The risk of new osteoporotic vertebral compression fractures in the year after percutaneous vertebroplasty. J Vas Interv Radiol. 2006;17: 71-6.

16. Trout AT, Kallmes DF, Kaufmann TJ. New fractures after vertebroplasty: adjacent fractures occur significantly sooner. AJNR. 2006:27:217-23. 